\title{
Determinants of altered intracellular endocrine-immune interplay in Bosnian war refugees suffering from PTSD
}

\author{
Kenneth P. Pitts, Ph.D. ${ }^{1}$, Ljiljana Joksimovic, M.D. ${ }^{2}$, Susann Steudte-Schmiedgen, Ph.D. ${ }^{3}$, \\ Nicolas Rohleder, Ph.D. ${ }^{1}$, Jutta M. Wolf, Ph.D. ${ }^{1, *}$ \\ ${ }^{1}$ Brandeis University, Waltham, MA 02453 USA \\ ${ }^{2}$ LVR-Klinikum Düsseldorf, 40629 Düsseldorf, Germany \\ ${ }^{3}$ Technische Universität Dresden, Dresden, Germany
}

*Jutta M. Wolf, Ph.D. ( ${ }^{\star}$ Corresponding author)

${ }^{1}$ Brandeis University, Psychology Department MS 062, 415 South St. Waltham, MA 02453 USA; E-mail: jmw@brandeis.edu; Phone: 001-781-736-3297; Fax: 001-781-736-3291

All authors declare they have no financial or other conflicts of interest. This work was supported by a Department of Defense SMART fellowship (K.P.P.) and a NARSAD Young Investigator Award (J.M.W.). 


\begin{abstract}
Posttraumatic Stress Disorder (PTSD) has been repeatedly linked to changes in glucocorticoid (GC) sensitivity. To increase our understanding of this phenomenon and its potential relevance for PTSD development and treatment, the current study investigates the interplay between two key moderators, glucocorticoid receptor (GRa) and GR co-chaperone FKBP5, and their relation to $\mathrm{GC}$ sensitivity.
\end{abstract}

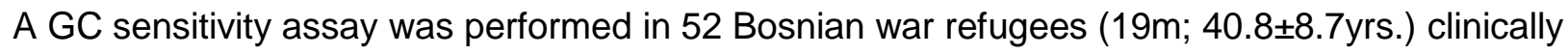
diagnosed with PTSD to divide the patient group into a high (HS) and a low (LS) GC sensitivity group. Expression of GRa and FKBP5 mRNA was quantified by real-time RT-PCR.

Links between gene expression and GC sensitivity were driven by the HS group of PTSD patients, which also showed increased expression of GRa but not FKBP5 compared to the LS group. Further, expressions of FKBP5 and GRa were strongly correlated in the HS patient group, while this association was missing in the LS PTSD group.

Our findings suggest that PTSD phenotypes may be characterized by differences in intracellular signaling transduction processes. The associations of expression of GRa and FKBP5 in the high-sensitive PTSD subgroup may thereby reflect physiological adaptation to preserve immune-relevant GC signaling. Further research is needed to understand the role and consequences of GRa-FKBP5 dissociation in low GC sensitivity PTSD patients.

Key words: posttraumatic stress disorder; glucocorticoid sensitivity; glucocorticoid receptor; FK506 binding protein 51; Bosnian war refugees 


\section{Introduction}

Posttraumatic Stress Disorder (PTSD) is a clinical condition that emerges as a possible consequence in response to trauma exposure. The etiology and maintenance of PTSD have frequently been linked to changes in endocrine and immune function, such as increased sympathetic reactivity and tone (Heim et al., 2000a; Heim and Nemeroff, 2009; Pallmeyer et al., 1986; Pitman et al., 1987; Wessa et al., 2006; Yehuda, 2001), dysregulation of the Hypothalamus-Pituitary-Adrenal (HPA) axis (Heim et al., 2000a; Heim and Nemeroff, 2009; Yehuda et al., 1991a), increased basal inflammation (Hoge et al., 2009; Maes et al., 1999; Rohleder et al., 2004; Spitzer et al., 2010), as well as the increased incidence of inflammationmediated diseases, like atherosclerosis (Pacella et al., 2013). With regards to HPA axis dysregulation most previous evidence suggests that PTSD is characterized by increased corticotropin-releasing hormone levels (Baker et al., 1999; Heim et al., 1997) and reduced cortisol levels (Heim et al., 2000a; Maes et al., 1999; Morris et al., 2012; Neylan et al., 2005; Steudte et al., 2013; Yehuda et al., 1991a), with hypocortisolemia presenting one pathway underlying the elevated inflammation often observed in PTSD patients (for a review see Pace and Heim, 2011).

Indeed, several studies support the notion of PTSD-related changes in the endocrine-immune interplay. For example, besides attenuated basal cortisol secretion, Rohleder et al. (2004) found an increased production of the pro-inflammatory marker interleukin-6 (IL-6) in response to lipopolysaccharide (LPS) challenge in Bosnian war refugees with PTSD compared to healthy controls. Furthermore, increased suppression of LPS-stimulated production of whole blood IL-6 and tumor necrosis factor-alpha (TNF- $\alpha$ ) by dexamethasone was observed in the PTSD group relative to controls. Similar results were reported for stimulated monoyctes as opposed to whole blood (de Kloet et al., 2006; Yehuda et al., 2002). As such, these findings suggest elevated glucocorticoid (GC) sensitivity as an organism's (unsuccessful) attempt to preserve cortisol 
inhibition of pro-inflammatory pathways (Wessa and Rohleder, 2007). Alternatively, it has been proposed that cortisol resistance in target tissues may be related to increased inflammation in tandem with hypocortisolism in this population (Fries et al., 2005; Heim et al., 2000b; Raison and Miller, 2003). It is important to highlight that evidence exists suggesting higher basal cortisol secretion (Steudte et al., 2011) or decreased sensitivity of immune cells in PTSD (de Kloet et al., 2007). While disparate findings may be a result of between-study differences in patient characteristics, such as timing, severity and frequency of traumatization, or disease comorbidities like major depression (Rohleder et al., 2010), they also emphasize the need to increase our understanding of the role of GC sensitivity in PTSD progression and its potential as a target for PTSD prevention and treatment.

In an attempt to elucidate factors contributing to alterations in GC sensitivity, previous research has investigated PTSD-related differences in mRNA expression for GR subtypes and GR target genes. GR subtypes have been proposed to modulate functional effects of cortisol as well as GR binding capacity (van Zuiden et al., 2013), however, findings suggesting a role for GR in PTSD are highly inconsistent. For example, O'Donovan et al. (2011) reported down-regulation of genes with response elements for GR subtypes in peripheral monocytes in male PTSD patients, suggesting cortisol resistance. Contrarily, other PTSD studies observed increased GR numbers in lymphocytes, pointing towards cortisol hypersensitivity (Yehuda et al., 1995; Yehuda et al., 1991b). Similarly, prospective studies by van Zuiden et al. (2011) revealed that high GR numbers in peripheral blood mononuclear cells (PBMCs) independently predicted PTSD symptom severity in soldiers after deployment to a combat zone. However, this was not accompanied by group differences in mRNA expression for GR subtypes (34). Lastly, some evidence suggests that increased intracellular GC responsiveness may occur independent of GR number density (Yehuda et al., 2004). Hence, the contradicting findings make it difficult to 
evaluate the relevance of the GR for PTSD etiology even though it is centrally involved in cortisol signaling (van Zuiden et al., 2013).

A further factor that has been considered to contribute to PTSD-related alterations in GC signaling is the FK506 binding protein 51 (FKBP5). FKBP5 is a gene-encoding co-chaperone of the GR heterocomplex and its expression is induced through a cortisol-initiated ultra-short negative feedback route (Grad and Picard, 2007). In more detail, subsequent to cortisol binding to the mature GR complex, FKBP5 disassociates, allowing FKBP4 to bind to the GR complex and thus its translocation to the nucleus (Wochnik et al., 2005). Consequently, increased FKBP5 expression is associated with relative cortisol resistance through decreased affinity of the GR with its substrate and by blocking nuclear translocation, thus reducing GR signaling (Binder, 2009; Yehuda et al., 2009). Conversely, an intrinsically low level of FKBP5 expression following trauma could facilitate increased GR signaling and provide a mechanism underlying the PTSD phenotype. Along these lines, reduced FKBP5 mRNA expression was observed in PTSD cohorts following the World Trade Center attacks in September 2001 (Yehuda et al., 2009) and low pre-deployment FKBP5 expression was predictive of PTSD symptoms after deployment (van Zuiden et al., 2012a). These findings were contradicted by Single Nucleotide Polymorphisms (SNPs) studies, which associated FKBP5 over-expression with peri-traumatic dissociation in medically injured children, a state that is known to predict later PTSD symptom development (Binder et al., 2008; Koenen and Uddin, 2010). Furthermore, SNPs for FKBP5 over-expression were linked to adult onset PTSD in individuals who suffered childhood abuse (Binder et al., 2008).

However, links of GR and FKBP5 expressions to functional assessments, particularly GC sensitivity of inflammation-related immune activation are still missing. Furthermore, given the interconnectedness of the processes, differences in GC sensitivity in PTSD may not be the result of changes in one of the two factors, but rather of changes in the interplay between GR 
and FKBP5 expression. In line with this notion, Mehta and colleagues (2011) argued that FKBP5 expression levels may differentiate between different subtypes of PTSD defined by, among others, the specific contribution of GC sensitivity to the disorder. Unfortunately, to date most studies assessed only one of the factors and the few studies assessing both did not consider their combined effect.

The present study thus aimed at addressing this issue by explicitly investigating the interplay between two of the key moderators of cortisol signaling, namely GRa and FKBP5 gene expression. Furthermore, we aimed at contrasting the individual with the combined effect of GR $\alpha$ and FKBP5 on cortisol-immune signaling assessed by GC sensitivity of IL-6 producing cells in whole blood.

Importantly, based on earlier findings suggesting GC sensitivity subtypes of PTSD, patients were categorized into high GC sensitive patients and low GC sensitive patients. This approach allowed determining the roles of GRa and FKBP5 in producing the range of cortisol sensitivity of stimulated cytokine production seen in PTSD, while acknowledging that disease-related deviation from healthy homeostatic processes may occur in a 'non-linear' manner (Rohleder et al., 2010). More specifically, GC sensitivity was considered a measure reflecting aggregated homeostatic adjustments that are aimed at compensating for disease-related endocrine or immune changes, as well as a measure reflecting adjustments that are no longer successful, but rather indicate dysfunctions resulting from too much wear and tear.

\section{Methods}

\subsection{Participants}

52 Bosnian war refugees (19 male) between the ages of 22 and $56(M=40.77, S D=8.7$ years) who were clinically diagnosed with PTSD according to DSM-IV criteria were recruited from the Psychosocial Center (PSC) of Refugees in Duesseldorf, Germany, a psychological treatment 
facility. The initial sample consisted of 57 PTSD patients, however, two individuals had to be excluded due to missing values, one for a Harvard Trauma Questionnaire (HTQ) score below cutoff of 2.5 , and two for being above cutoff of $Z \pm 3$ for expression of $G R \alpha$ or $G C$ sensitivity. PTSD patients had HTQ scores of $M=3.88$ (SD: 0.11) and among the most frequently reported types of trauma (experienced or witnessed) were combat situations $(n=49)$, homelessness $(n=44)$, and murder of family member ( $n=39$; see Table 1 for more details).

Table 1: Frequency of traumatic life events experienced or witnessed by PTSD patients.

\begin{tabular}{lccc}
\hline & Experienced & Witnessed & Total \\
\cline { 2 - 4 } Combat Situations & 47 & 2 & 49 \\
Homelessness & 42 & 2 & 44 \\
Brain Wash & 39 & 4 & 43 \\
Missing & 39 & 4 & 43 \\
Murder of Family Member & 31 & 8 & 39 \\
Unnatural Death of Friend or Family & 31 & 6 & 37 \\
Rape & 27 & 11 & 38 \\
Lack of Food & 26 & 13 & 39 \\
Isolation & 26 & 8 & 34 \\
Separation from Family & 22 & 17 & 39 \\
Murder of Unknown Person & 21 & 12 & 33 \\
Near Death Experience & 19 & 18 & 37 \\
Injuries & 18 & 5 & 23 \\
Torture & 15 & 15 & 30 \\
Captivity & 13 & 7 & 20 \\
No Medical Care Access & 7 & 20 & 27 \\
\hline On average, paricapars experienced & & & \\
\hline
\end{tabular}

Note. On average, participants experienced 8.81 traumata $(S D=3.5 ; \min =1$, $\max =15)$ and witnessed 3.53 traumata $(S D=2.4 ; m i=1, \max =10)$. Types of trauma are listed according to frequency experienced. 
To allow qualitative comparisons, a reference group of 16 healthy age- and gender-matched German individuals were assessed in parallel (7 males; age: $M=45.06, S D=9.67$ years; $F(1,65)=2.71, p=.11)$. The study protocol was approved by the ethics committee of the University of Duesseldorf, Germany. Data collection occurred between November 2002 and April 2003.

\subsection{Procedures}

Interested patients were assessed (LJ) and included in the PTSD group if they met DSM-IV diagnostic criteria for PTSD and if they obtained a minimum score of 2.5 on the HTQ. Patients who reported physical illness, medication prescriptions, or substance abuse were excluded from this study. Furthermore, other current or past psychiatric or medical disorders were assessed with the German version of the revised Symptom Checklist-90 (SCL-90-R; Derogatis and Cleary, 1977). German participants were screened on the phone for current or past psychiatric or medical disorders and only those reporting no psychiatric or health problems and being medication free were included in the healthy reference group. Medical history was further confirmed by a physician-lead in-person interview upon arrival in the laboratory.

Eligible participants were assessed at the PSC (PTSD cohort) or the laboratory (controls) between 9 AM and 1 PM. All participants were informed orally and in writing (in German or by Bosnian translation) about the aims and methods of the study prior to obtaining written consent. After obtaining consent, participants provided basic demographic information and a trained phlebotomist collected venous blood samples. More specifically, a total of $10.2 \mathrm{~mL}$ blood was collected in one $5 \mathrm{~mL}$ heparinized syringe, one $2.5 \mathrm{~mL}$ EDTA Monovette, and one $2.7 \mathrm{~mL}$ EDTA Monovette. Patients' therapist was present for support during blood collection. After blood collection, all participants were paid and patients entered an outpatient treatment program. 


\subsection{Measures}

After collection, all patient blood samples were stored at room temperature for a standard 90 minutes before further processing to avoid variation caused by different transportation durations.

\subsubsection{Glucocorticoid sensitivity assay.}

The ex vivo GC sensitivity assay was performed from venous blood collected in $5 \mathrm{~mL}$ heparinized syringes (Braun, Melsungen, Germany) during regular support group meetings for the PTSD patients and during laboratory visits for the healthy participants) according to established protocols (e.g., Rohleder et al., 2001). In more detail, after arriving at the laboratory, the sample of heparinized whole blood was diluted 10:1 with saline and then split between six 400- $\mu \mathrm{L}$ aliquots in a 24-well cell culture plate (Greiner, Nuertingen, Germany). Cytokine production was stimulated by adding the bacterial endotoxin lipopolysaccharide (LPS, Escherichia coli, Sigma, Deisenhofen, Germany) while co-incubating with dexamethasone (DEX) resulting in $30 \mathrm{ng} / \mathrm{mL}$ LPS final concentration in all wells and $0,10^{-9}, 10^{-8}, 5^{\star} 10^{-8}, 10^{-7}$, and $10^{-6}$ DEX across the six wells. Plates were then incubated for six hours at $37^{\circ} \mathrm{C}$ and $5 \%$ $\mathrm{CO}^{2}$. After incubation, the plates were centrifuged at $2000 \mathrm{~g}$ for 10 minutes at $4^{\circ} \mathrm{C}$. Plasma supernatant was stored at $-80^{\circ} \mathrm{C}$ until the analysis was conducted at the end of the study. In line with the GC sensitivity literature and the cytokine's central role in health-relevant immune activation and inflammatory processes, interleukin-6 (IL-6) concentrations were measured by commercially available enzyme linked immunosorbent assay (ELISA; BD Pharmingen, San Diego, California) with a detection limit at $4.7 \mathrm{pg} / \mathrm{mL}$. Sample absorbance (Anthos HTII, Anthos Labtec, Salzburg, Austria) was transformed to IL-6 concentration (ng/mL) using a standard curve computed by Anthos Winread 2.3 software (Anthos Labtec). Next, IL-6 levels were corrected for number of monocytes in the sample and expressed as ng $/ 10^{6}$ monocytes. Monocyte numbers were thereby determined from blood collected in $2.7 \mathrm{~mL}$ EDTA monovettes (Sarstedt, Rommelsdorf, Germany). In more detail, each EDTA sample was measured 5 times 
using an AcT Diff Cell counter (Beckman-Coulter, Krefeld, Germany) and average numbers of monocytes were computed for each sample. Lastly, the $50 \%$ inhibitory concentration (IC $\left.{ }_{50}\right)$ for each individual dose-response curve for DEX inhibition of maximum LPS-induced IL-6 production was calculated as an index inversely related to GC sensitivity.

\subsubsection{MRNA Expression Assay.}

Leukocyte expression of glucocorticoid receptor (GRa) mRNA and FKBP5 mRNA was quantified by real-time reverse transcriptase - polymerase chain reaction (RT-PCR). In more detail, RNA was extracted from 1.5ml blood collected in EDTA monovettes (Sarstedt, Rommelsdorf, Germany) by QIAamp RNA Blood Mini Kit (Qiagen, Hilden, Germany) and stored at -80C. After study completion, one-step RT-PCR assays based on 5-nuclease activity of 6carboxyfluorescein-labeled TaqMan probes (TaqMan One-Step RT-PCR: Applied Biosystems), were conducted on a Applied Biosystems Prism 7000 Sequence Detection System. The GRa primer sequence was developed by Applied Biosystems based on consensus of the National Center for Biotechnology Information RefSeq, NM_000176 (forward:

AGTGGTTGAAAATCTCCTTAACTATTGCT; reverse: GGTATCTGATTGGTGATGATTTCAGCTA). For FKBP5, a commercial TaqMan® Gene Expression Assay kit was used (Hs00188025_m1). Levels of 18S mRNA were quantified in parallel as the internal control (TaqMan® Gene Expression Assay kit \#Hs99999901). All assays were run in duplicates and repeated if indicated.

The thermal cycling included 30 minutes of reverse transcription at $48^{\circ} \mathrm{C}$, followed by 10 minutes of Taq enzyme activation at $95^{\circ} \mathrm{C}$ followed by 40 cycles of amplification, which consisted of 15 seconds of denaturing at $95^{\circ} \mathrm{C}$ and then 60 seconds of annealing at $60^{\circ} \mathrm{C}$. SDS 1.1 RQ software (Applied Biosystems) was used to establish threshold cycle $\left(\mathrm{C}_{\mathrm{T}}\right)$ numbers. The data were normalized by subtracting $\mathrm{C}_{\mathrm{T}}$ internal control from $\mathrm{C}_{\mathrm{T}}$ target gene. 
To determine mRNA expression values for analysis, relative quantities were derived by subtracting each mRNA expression value from the highest value within its category to correct for initial concentrations and to align positive standardized values ( $Z$ scores) with higher mRNA concentrations. Higher relative quantities indicate greater expression of the target genes, with each unit difference indicating a two-fold difference in expression.

\subsection{Analytical Plan}

All analyses were performed in SPSS version 21 and controlled for age and gender. Preliminary analyses were conducted comparing healthy participants and PTSD patients. More specifically, to replicate the finding of increased cortisol sensitivity in PTSD, IC $_{50}$ scores were compared between healthy and PTSD participants by 2-way ANCOVA. This analysis was repeated for FKBP5 and GRa mRNA expression. Lastly, partial correlations were computed to assess associations between FKBP5 and GRa mRNA expression vectors in healthy as well as PTSD participants.

To address the study's main aims, first the PTSD group was divided into a high cortisol sensitive group (HiSens) and a low cortisol sensitive group (LoSens). Since research supporting the definition of a cut-of point indicating a switch from homeostatic to dysfunctional endocrineimmune processes is lacking, groups were formed by $\mathrm{IC}_{50}$ median split. A 2-way ANCOVA was computed to compare $\mathrm{IC}_{50}$ scores between the HiSens and LoSens subgroups to confirm that the median split produced two significantly distinct groups. Next, partial correlations were computed to assess associations between FKBP5 and GRa mRNA expression vectors in HiSens and LoSens patients. Lastly, to assess the individual versus combined role of FKBP5 and GRa mRNA expression vectors in cortisol sensitivity, hierarchical regression analyses were computed. After entering age and gender, GRa (model 1a) and FKBP5 (model 1b) were regressed on $\mathrm{IC}_{50}$ in separate analyses to ascertain the predictive value of each vector alone on cortisol sensitivity. Next, GRa and FKBP5 were regressed together on IC 50 (model 2) to assess 
each factor's effect above and beyond the effect of the other, and finally, the interaction term FKBP5*GRa (model 3) was added to the regression to test for moderation effects. P-values < .05 were considered indicative of significant differences or associations and increases in $p$ values across regression analysis models were interpreted as indicative of mediation effects. Changes in $\mathrm{R}^{2}$ and partial $\eta^{2}$ are reported as effect sizes and differences between correlations are tested using Fisher's r-to-z transformation.

\section{Results}

\subsection{Preliminary analyses}

As predicted, PTSD patients showed significantly higher GC sensitivity (lower IC ${ }_{50}$ ) of IL-6 producing cells in whole blood $\left(F(1,64)=20.82, p<.001, p \eta^{2}=.25\right)$ than healthy participants. Furthermore, a trend for higher $G R a$ expression $\left(F(1,64)=3.71, p=.059, \eta_{p} \eta^{2}=.06\right)$ as well as significantly higher FKBP5 expression levels $\left(F(1,64)=38.41, p<.001,{ }_{p} \eta^{2}=.38\right)$ were observed when compared to healthy participants. Interestingly, while expressions of FKBP5 and GRa were positively correlated in the healthy group $\left(r_{12}=.59, p=.027\right)$, they were uncorrelated in PTSD patients $\left(r_{48}=.20, p=.17 ; z=1.52, p=.13\right)$.

\subsection{Gene expression in high and low GC sensitive PTSD subgroups}

Using a median split on $\mathrm{IC}_{50}$ levels of PTSD patients resulted in two distinct groups in terms of GC sensitivity $\left(\mathrm{IC}_{50}: \mathrm{F}(1,48)=75.50, \mathrm{p}<.001, \mathrm{p}^{2}=.61\right.$; Fig. 1$)$. Importantly, this was not accompanied by differences in patients' initial immune response to challenge (LPS-stimulated IL-6 production: $\left.F(1,48)=1.67, \mathrm{p}=.20, \mathrm{p} \eta^{2}=.03\right)$.

Furthermore, the high GC sensitive PTSD subgroup expressed significantly more GRa mRNA than the low $G C$ sensitive group $\left(F(1,48)=6.47, p=.014\right.$, $p \eta^{2}=.12 ;$ Fig. 2$)$, while no significant differences between PTSD subgroups were found in FKBP5 mRNA expression $(F(1,48)=1.00$, $\mathrm{p}=.32, \mathrm{p} \eta^{2}=.02 ;$ Fig. 2). 
Figure 1: Increased GC sensitivity as indicated by inhibitory concentration 50 (IC 50 , unit: 10-8 Mol DEX) of stimulated IL-6 production in PTSD patients compared to healthy individuals.

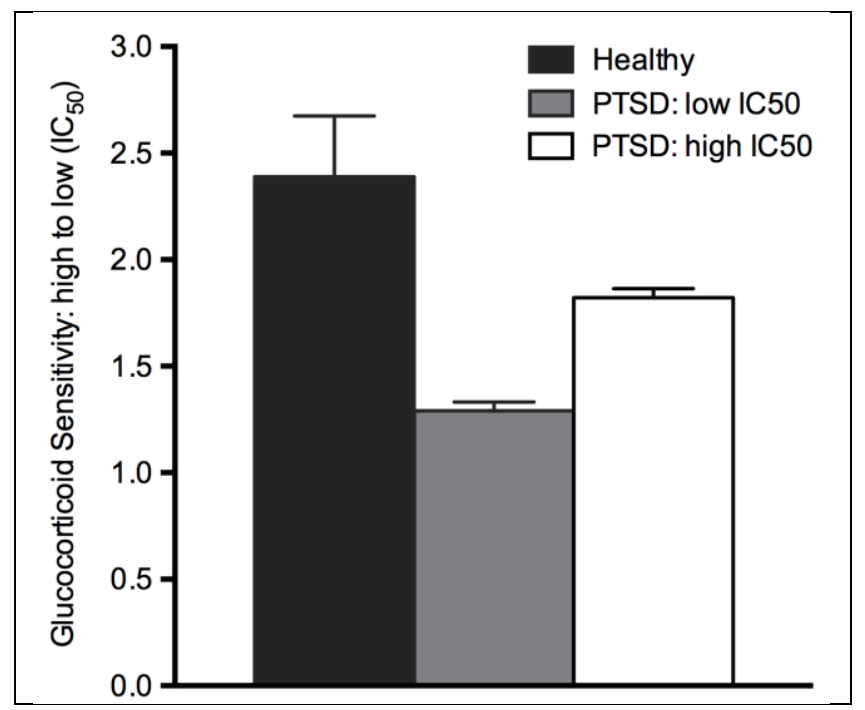

However, FKBP5 and GRa expression were highly correlated in the high GC sensitive PTSD subgroup $\left(r_{22}=.66, p=.001\right)$, yet this was not evident in the low $G C$ sensitive PTSD subgroup $\left(r_{22}=-.18, p=.40 ; z=3.31, p<.001 ;\right.$ Fig. 3$)$.

Figure 2: GRa expression (left y-axis) in PTSD patients with high GC sensitivity (low IC $\left.C_{50}\right)$ differed from GRa expression in healthy individuals and PTSD patients with low GC sensitivity (high $I C_{50}$ ). Contrarily, FKBP5 expression (right y-axis) did not differ between the two patient groups, but between patients overall and healthy individuals.




Figure 3: Positive associations between GRa and FKBP5 expression levels were observed for healthy participants (black dots, black solid line) as well as PTSD patients with high GC sensitivity (low IC 50; gray dots, gray solid line). No association in GRa and FKBP5 expression levels was found for PTSD patients with low GC sensitivity (high IC 50; white squares, dashed line).

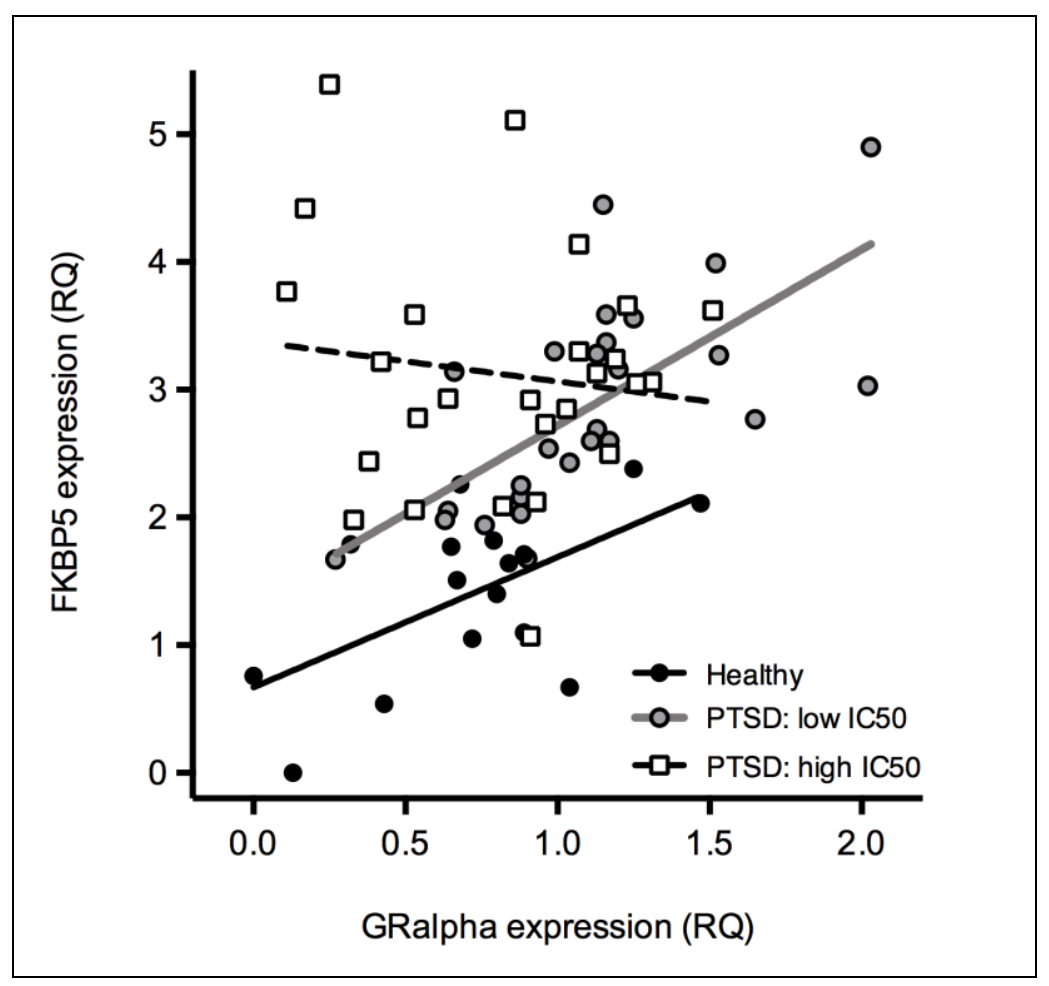

Assessing relationships between mRNA expressions and GC sensitivity within each of the PTSD subgroups revealed no GRa relationship with cortisol sensitivity in either subgroup, neither by itself nor when controlling for FKPB5 (all $p \geq .09$, all $\delta R^{2}<.11$ ). However, FKBP5 expression levels showed a significant positive relationship with GC sensitivity (i.e., a negative relationship with $\left.\mathrm{IC}_{50}\right)$ in the high $\mathrm{GC}$ sensitive subgroup only $(\mathrm{B}=-0.12, \mathrm{SE}=0.05$, beta $=-.47$, $p=.023, \delta R^{2}=.20$; low $G C$ sensitive group: $\left.p=.21, \delta R^{2}=.05\right)$. This effect was reduced when controlling for $\mathrm{GR} \alpha$ effects (FKBP5: $\mathrm{B}=-0.11, \mathrm{SE}=0.07$, beta=-.41, $\mathrm{p}=.13, \delta \mathrm{R}^{2}=.003$ ), suggesting some shared variance between GRa and GC sensitivity contributing indirectly to effects of FKBP5 on GC sensitivity. In neither group did the interaction of GRa and FKBP5 predict GC sensitivity levels (p's>.20, all $\delta R^{2}<.05$ ). 
Of note, GRa and FKBP5 expressions in healthy participants were neither individually nor in combination related to $\mathrm{GC}$ sensitivity (all $\mathrm{p}>.41$, all $\delta \mathrm{R}^{2}<.06$ ). Furthermore, $\mathrm{PTSD}$ severity as measured by HTQ was considered as a factor explaining some of the differences between the high and low GC sensitive subgroups. However, no group differences in HTQ scores were found $\left(F(1,46)=0.01, p=.92, p \eta^{2}<.001\right)$ and rerunning all analyses controlling for HTQ scores did not change the pattern of results reported above. The same was true for psychological problems and symptoms of psychopathology assessed by the revised Symptom Checklist-90 (SCL-90-R; global severity index: mean=2.03, $\mathrm{SD}=0.78$; high versus low $\mathrm{GC}$ sensitivity subgroups: $\left.F(1,46)=0.09, p=.77, p \eta^{2}=.002\right)$.

\section{Discussion}

In summary, the current study confirmed previous findings of increased GC sensitivity of inflammatory cytokine production in whole blood in PTSD. Furthermore, PTSD was in general associated with increased mRNA expression of both GRa and FKBP5. However, when assessing PTSD subtypes, elevated gene expression levels as well as highly correlated mRNA expression of GRa and FKBP5 were observed in the high GC sensitive PTSD group only. In contrast, patients in the low GC sensitive group showed reduced GRa mRNA expression, which further was unrelated to FKBP5 mRNA expression.

Our finding of enhanced suppression of LPS-induced production of IL-6 in whole blood by dexamethasone supports prior reports of increased GC sensitivity as a major component of the PTSD phenotype (Rohleder et al., 2004; Rohleder et al., 2010; van Zuiden et al., 2012b; Yehuda, 2001; Yehuda et al., 1995; Yehuda et al., 2004). Increased GC sensitivity has been described together with hypocortisolemia (Daskalakis et al., 2013; Rohleder et al., 2004; Yehuda et al., 1995), which is the putative cause of increased inflammation observed in PTSD (O'Donovan et al., 2015; Pace et al., 2012; Spitzer et al., 2010). This model describes an immune system that adapts to persistently low cortisol by increasing GC sensitivity of immune 
cells to recover the immune-inhibitory cortisol signal (Rohleder et al., 2010). In this way, immune system activity remains linked to HPA axis output, even under PTSD associated hypocortisolemia. However, while an intriguing speculation, given that PTSD is still associated with increased inflammatory activity, this raises the question whether the observed increase in GC sensitivity of immune cells is either not effective in restoring cortisol effects in the long term or rather a secondary and unsuccessful attempt of immune self-inhibition.

To examine the role of cortisol signaling in this process in more depth, the present study focused on two key modulating factors that have been targeted by recent PTSD research: GR $\alpha$ and FKBP5. For both factors, the current study revealed marked expression upregulation in PTSD patients. While the finding for GRa upregulation is in accordance with the majority of previous research suggesting a higher GR number in PTSD patients (van Zuiden et al., 2012a; van Zuiden et al., 2011), it is at variance with research suggesting a down-regulation of GR target genes in peripheral monocytes in male PTSD patients or no group differences in GR mRNA expression levels (34). The current observation of FKBP5 upregulation, further, is in contrast to earlier research describing its downregulation in PTSD (Yehuda et al., 2009). This discrepancy could in part be the result of differences in type and duration of trauma between the studies' subject populations. For example, the study by Yehuda et al. (40) assessed 9-11 survivors while the current population consisted of war refugees who were exposed to multiple traumatic events lasting for days to months followed by years of disenfranchisement potentially reinforcing negative war-time experiences. The differences in temporal and psychological characteristics of the precipitating trauma between studies, as well as the current participants' chronic identification as refugees may induce specific physiological burdens contributing to differentiated expression of GC signaling factors, including FKBP5, and to altered signaling dynamics. This explanation is supported by a complex view of PTSD etiology that suggests an array of alterations in cognitive and physiological parameters during the development of the 
disorder (Badour and Feldner, 2013; McLean et al., 2014; Roth et al., 1997). Thus, to acknowledge these differential dynamics, the current study assessed PTSD subtypes based on patients GC sensitivity (Mehta et al., 2011).

Importantly, earlier research efforts considered FKBP5 and GRa independently as negative or positive, respectively, mediators of the GC signal. However, the present study tested if statistical relationships exist 1) between these factors and 2) with GC sensitivity. In terms of the former, we found that while GRa and FKBP5 were highly correlated in the healthy population, no association between these factors was observed in the PTSD group, indicating a linked expression only under non-PTSD physiological conditions. To explore these differential dynamics further, gene expression associations were compared between patients exhibiting higher GC sensitivity and patients exhibiting lower GC sensitivity. Interestingly, similar to the healthy individuals, mRNA expression of GRa and FKBP5 was highly correlated in the high sensitive subgroup, yet no relationship existed between these two variables in the low GC sensitive subgroup. This observation was accompanied by significantly higher GRa expression levels in the high GC sensitive PTSD group compared to the low GC sensitive PTSD group. It could be speculated that increased expression of GRa in concert with increased expression of FKBP5 would help preserve the immune system's sensitivity to cortisol. Contrarily, in the low sensitive PTSD subgroup, desynchronized increased FKBP5 expression and comparatively reduced GRa expression may contribute to a reduced sensitivity of the immune system to a suppressive cortisol signal. Assuming consistent immunogenic activity across the three investigated groups, the low sensitive PTSD group would be expected to have increased inflammatory tone and potentially higher incidence of inflammation-mediated diseases than the healthy and high sensitive groups.

This interpretation is to some extent supported by the observation that for PTSD patients in the high GC sensitivity group only, GRa mediated the link between FKBP5 and GC sensitivity. 
Contrarily, although interlinked as well, neither GRa nor FKBP5 alone or in combination predicted GC sensitivity in healthy participants. These findings emphasize the importance of GRa and FKBP5 parity in preserving immune-relevant GC signaling under presumably hypocortisolemic conditions in PTSD. It also draws additional attention to the lack of association of these factors with each other and with GC sensitivity in the low sensitive subgroup. It is unclear at this point if a GRa-FKBP5 desynchronization in this group of patients represents a first step in an unraveling endocrine-immune communication or a transition stage allowing immune adjustments necessary to counterbalance disease-related endocrine changes.

Of note and given the study's focus on differential processes within the PTSD patient group, German participants were assessed as a reference group only. Despite power issues related to the small sample size, heritable, cultural, and situational differences discourage interpretation of statistical differences between the two groups, as these factors may promote between-group differences in stress system homeostasis that are unrelated to PTSD status per se. Similarly, choosing a non-traumatized population does not allow to decide whether observed differences are due to trauma exposure or PTSD. Thus, future studies will have to determine whether the current findings in healthy German participants can be replicated in a larger healthy Bosnian population exposed to trauma. Furthermore, the focus of the current study was on determinants of the altered intracellular endocrine-immune interplay observed in PTSD patients along the cortisol signaling pathway. Future studies may expand on the present findings by assessing the role of determinants along immune pathways as well as peripheral endocrine and immune markers, such as basal cortisol and plasma IL-6.

In summary, our findings support the role of increased GC sensitivity as a major component of the PTSD phenotype. They further illustrate that PTSD is a heterogeneous condition suggesting a need for research that simultaneously considers the effect of multiple pathways in the progression of the PTSD phenotype. By assessing alterations in mRNA expression along 
multiple pathways, intracellular processes that support the phenotype can be reflected upon the temporal and qualitative characteristics of the trauma and subsequent stress. In this way, a mechanistic description of disease progression and functionally relevant interventions to affect the course of the disorder can be developed. 


\section{Acknowledgements}

We would like to thank all participants as well as the Psychosocial Center (PSC) of Refugees in Duesseldorf, Germany, for supporting this study. 


\section{References}

Badour, C.L., Feldner, M.T., 2013. Trauma-related reactivity and regulation of emotion: associations with posttraumatic stress symptoms. Journal of behavior therapy and experimental psychiatry $44,69-76$.

Baker, D.G., West, S.A., Nicholson, W.E., Ekhator, N.N., Kasckow, J.W., Hill, K.K., Bruce, A.B., Orth, D.N., Geracioti, T.D., Jr., 1999. Serial CSF corticotropin-releasing hormone levels and adrenocortical activity in combat veterans with posttraumatic stress disorder. Am J Psychiatry 156, 585-588.

Binder, E.B., 2009. The role of FKBP5, a co-chaperone of the glucocorticoid receptor in the pathogenesis and therapy of affective and anxiety disorders. Psychoneuroendocrinology 34 Suppl 1, S186-195.

Binder, E.B., Bradley, R.G., Liu, W., Epstein, M.P., Deveau, T.C., Mercer, K.B., Tang, Y., Gillespie, C.F., Heim, C.M., Nemeroff, C.B., Schwartz, A.C., Cubells, J.F., Ressler, K.J., 2008. Association of FKBP5 polymorphisms and childhood abuse with risk of posttraumatic stress disorder symptoms in adults. Jama 299, 1291-1305.

Daskalakis, N.P., Lehrner, A., Yehuda, R., 2013. Endocrine aspects of post-traumatic stress disorder and implications for diagnosis and treatment. Endocrinology and metabolism clinics of North America 42, 503-513.

de Kloet, C.S., Vermetten, E., Bikker, A., Meulman, E., Geuze, E., Kavelaars, A., Westenberg, H.G., Heijnen, C.J., 2007. Leukocyte glucocorticoid receptor expression and immunoregulation in veterans with and without post-traumatic stress disorder. Mol Psychiatry 12, 443-453. 
de Kloet, C.S., Vermetten, E., Geuze, E., Kavelaars, A., Heijnen, C.J., Westenberg, H.G., 2006. Assessment of HPA-axis function in posttraumatic stress disorder: pharmacological and non-pharmacological challenge tests, a review. J Psychiatr Res 40, 550-567.

Derogatis, L.R., Cleary, P.A., 1977. Factorial invariance across gender for the primary symptom dimensions of the SCL-90. The British journal of social and clinical psychology 16, 347356.

Fries, E., Hesse, J., Hellhammer, J., Hellhammer, D.H., 2005. A new view on hypocortisolism. Psychoneuroendocrinology 30, 1010-1016.

Grad, I., Picard, D., 2007. The glucocorticoid responses are shaped by molecular chaperones. Mol Cell Endocrinol 275, 2-12.

Heim, C., Ehlert, U., Hellhammer, D.H., 2000a. The potential role of hypocortisolism in the pathophysiology of stress-related bodily disorders. Psychoneuroendocrinology 25, 1-35.

Heim, C., Nemeroff, C.B., 2009. Neurobiology of posttraumatic stress disorder. CNS spectrums 14, 13-24.

Heim, C., Newport, D.J., Miller, A.H., Nemeroff, C.B., 2000b. Long-term neuroendocrine effects of childhood maltreatment. Jama 284, 2321.

Heim, C., Owens, M.J., Plotsky, P.M., Nemeroff, C.B., 1997. Persistent changes in corticotropinreleasing factor systems due to early life stress: relationship to the pathophysiology of major depression and post-traumatic stress disorder. Psychopharmacology bulletin 33, 185-192.

Hoge, E.A., Brandstetter, K., Moshier, S., Pollack, M.H., Wong, K.K., Simon, N.M., 2009. Broad spectrum of cytokine abnormalities in panic disorder and posttraumatic stress disorder. Depress Anxiety 26, 447-455. 
Koenen, K.C., Uddin, M., 2010. FKBP5 polymorphisms modify the effects of childhood trauma. Neuropsychopharmacology : official publication of the American College of Neuropsychopharmacology 35, 1623-1624.

Maes, M., Lin, A.H., Delmeire, L., Van Gastel, A., Kenis, G., De Jongh, R., Bosmans, E., 1999. Elevated serum interleukin-6 (IL-6) and IL-6 receptor concentrations in posttraumatic stress disorder following accidental man-made traumatic events. Biol Psychiatry 45, 833839.

McLean, C.P., Morris, S.H., Conklin, P., Jayawickreme, N., Foa, E.B., 2014. Trauma Characteristics and Posttraumatic Stress Disorder among Adolescent Survivors of Childhood Sexual Abuse. Journal of family violence 29, 559-566.

Mehta, D., Gonik, M., Klengel, T., Rex-Haffner, M., Menke, A., Rubel, J., Mercer, K.B., Putz, B., Bradley, B., Holsboer, F., Ressler, K.J., Muller-Myhsok, B., Binder, E.B., 2011. Using polymorphisms in FKBP5 to define biologically distinct subtypes of posttraumatic stress disorder: evidence from endocrine and gene expression studies. Arch. Gen. Psychiatry 68, 901-910.

Morris, M.C., Compas, B.E., Garber, J., 2012. Relations among posttraumatic stress disorder, comorbid major depression, and HPA function: a systematic review and meta-analysis. Clinical psychology review 32, 301-315.

Neylan, T.C., Brunet, A., Pole, N., Best, S.R., Metzler, T.J., Yehuda, R., Marmar, C.R., 2005. PTSD symptoms predict waking salivary cortisol levels in police officers. Psychoneuroendocrinology 30, 373-381.

O'Donovan, A., Chao, L.L., Paulson, J., Samuelson, K.W., Shigenaga, J.K., Grunfeld, C., Weiner, M.W., Neylan, T.C., 2015. Altered inflammatory activity associated with reduced 
hippocampal volume and more severe posttraumatic stress symptoms in Gulf War veterans. Psychoneuroendocrinology 51, 557-566.

O'Donovan, A., Sun, B., Cole, S., Rempel, H., Lenoci, M., Pulliam, L., Neylan, T., 2011. Transcriptional control of monocyte gene expression in post-traumatic stress disorder. Disease markers 30, 123-132.

Pace, T.W., Heim, C.M., 2011. A short review on the psychoneuroimmunology of posttraumatic stress disorder: from risk factors to medical comorbidities. Brain, behavior, and immunity $25,6-13$.

Pace, T.W., Wingenfeld, K., Schmidt, I., Meinlschmidt, G., Hellhammer, D.H., Heim, C.M., 2012. Increased peripheral NF-kappaB pathway activity in women with childhood abuserelated posttraumatic stress disorder. Brain, behavior, and immunity 26, 13-17.

Pacella, M.L., Hruska, B., Delahanty, D.L., 2013. The physical health consequences of PTSD and PTSD symptoms: a meta-analytic review. Journal of anxiety disorders $27,33-46$.

Pallmeyer, T.P., Blanchard, E.B., Kolb, L.C., 1986. The psychophysiology of combat-induced post-traumatic stress disorder in Vietnam veterans. Behaviour research and therapy 24, 645-652.

Pitman, R.K., Orr, S.P., Forgue, D.F., de Jong, J.B., Claiborn, J.M., 1987. Psychophysiologic assessment of posttraumatic stress disorder imagery in Vietnam combat veterans. Archives of general psychiatry 44, 970-975.

Raison, C.L., Miller, A.H., 2003. When not enough is too much: the role of insufficient glucocorticoid signaling in the pathophysiology of stress-related disorders. Am J Psychiatry 160, 1554-1565. 
Rohleder, N., Joksimovic, L., Wolf, J.M., Kirschbaum, C., 2004. Hypocortisolism and increased glucocorticoid sensitivity of pro-Inflammatory cytokine production in Bosnian war refugees with posttraumatic stress disorder. Biological psychiatry 55, 745-751.

Rohleder, N., Schommer, N.C., Hellhammer, D.H., Engel, R., Kirschbaum, C., 2001. Sex differences in glucocorticoid sensitivity of proinflammatory cytokine production after psychosocial stress. Psychosom Med 63, 966-972.

Rohleder, N., Wolf, J.M., Wolf, O.T., 2010. Glucocorticoid sensitivity of cognitive and inflammatory processes in depression and posttraumatic stress disorder. Neuroscience and biobehavioral reviews 35, 104-114.

Roth, S., Newman, E., Pelcovitz, D., van der Kolk, B., Mandel, F.S., 1997. Complex PTSD in victims exposed to sexual and physical abuse: results from the DSM-IV Field Trial for Posttraumatic Stress Disorder. Journal of traumatic stress 10, 539-555.

Spitzer, C., Barnow, S., Volzke, H., Wallaschofski, H., John, U., Freyberger, H.J., Lowe, B., Grabe, H.J., 2010. Association of posttraumatic stress disorder with low-grade elevation of C-reactive protein: evidence from the general population. Journal of psychiatric research 44, 15-21.

Steudte, S., Kirschbaum, C., Gao, W., Alexander, N., Schonfeld, S., Hoyer, J., Stalder, T., 2013. Hair cortisol as a biomarker of traumatization in healthy individuals and posttraumatic stress disorder patients. Biological psychiatry 74, 639-646.

Steudte, S., Kolassa, I.T., Stalder, T., Pfeiffer, A., Kirschbaum, C., Elbert, T., 2011. Increased cortisol concentrations in hair of severely traumatized Ugandan individuals with PTSD. Psychoneuroendocrinology 36, 1193-1200.

van Zuiden, M., Geuze, E., Willemen, H.L., Vermetten, E., Maas, M., Amarouchi, K., Kavelaars, A., Heijnen, C.J., 2012a. Glucocorticoid receptor pathway components predict 
posttraumatic stress disorder symptom development: a prospective study. Biological psychiatry $71,309-316$.

van Zuiden, M., Geuze, E., Willemen, H.L., Vermetten, E., Maas, M., Heijnen, C.J., Kavelaars, A., 2011. Pre-existing high glucocorticoid receptor number predicting development of posttraumatic stress symptoms after military deployment. The American journal of psychiatry 168, 89-96.

van Zuiden, M., Heijnen, C.J., Maas, M., Amarouchi, K., Vermetten, E., Geuze, E., Kavelaars, A., 2012b. Glucocorticoid sensitivity of leukocytes predicts PTSD, depressive and fatigue symptoms after military deployment: A prospective study. Psychoneuroendocrinology 37, $1822-1836$.

van Zuiden, M., Kavelaars, A., Geuze, E., Olff, M., Heijnen, C.J., 2013. Predicting PTSD: preexisting vulnerabilities in glucocorticoid-signaling and implications for preventive interventions. Brain, behavior, and immunity 30, 12-21.

Wessa, M., Rohleder, N., 2007. Endocrine and inflammatory alterations in posttraumatic stress disorder. Expert Rev Endocrinol Metabol 2, 91-122.

Wessa, M., Rohleder, N., Kirschbaum, C., Flor, H., 2006. Altered cortisol awakening response in posttraumatic stress disorder. Psychoneuroendocrinology 31, 209-215.

Wochnik, G.M., Ruegg, J., Abel, G.A., Schmidt, U., Holsboer, F., Rein, T., 2005. FK506-binding proteins 51 and 52 differentially regulate dynein interaction and nuclear translocation of the glucocorticoid receptor in mammalian cells. J Biol Chem 280, 4609-4616.

Yehuda, R., 2001. Biology of posttraumatic stress disorder. The Journal of clinical psychiatry 62 Suppl 17, 41-46.

Yehuda, R., Boisoneau, D., Lowy, M.T., Giller, E.L., Jr., 1995. Dose-response changes in plasma cortisol and lymphocyte glucocorticoid receptors following dexamethasone 
administration in combat veterans with and without posttraumatic stress disorder. Arch Gen Psychiatry 52, 583-593.

Yehuda, R., Cai, G., Golier, J.A., Sarapas, C., Galea, S., Ising, M., Rein, T., Schmeidler, J., Muller-Myhsok, B., Holsboer, F., Buxbaum, J.D., 2009. Gene expression patterns associated with posttraumatic stress disorder following exposure to the World Trade Center attacks. Biological psychiatry 66, 708-711.

Yehuda, R., Giller, E.L., Southwick, S.M., Lowy, M.T., Mason, J.W., 1991a. Hypothalamicpituitary-adrenal dysfunction in posttraumatic stress disorder. Biological psychiatry 30, 1031-1048.

Yehuda, R., Golier, J.A., Yang, R.K., Tischler, L., 2004. Enhanced sensitivity to glucocorticoids in peripheral mononuclear leukocytes in posttraumatic stress disorder. Biol Psychiatry $55,1110-1116$.

Yehuda, R., Halligan, S.L., Grossman, R., Golier, J.A., Wong, C., 2002. The cortisol and glucocorticoid receptor response to low dose dexamethasone administration in aging combat veterans and holocaust survivors with and without posttraumatic stress disorder. Biol Psychiatry 52, 393-403.

Yehuda, R., Lowy, M.T., Southwick, S.M., Shaffer, D., Giller, E.L., Jr., 1991b. Lymphocyte glucocorticoid receptor number in posttraumatic stress disorder. Am J Psychiatry 148, 499-504. 